\title{
Changes in Baroreflex Sensitivity in Head-Down Tilt Position
}

\author{
Junko Nakahira", Shoko Nakano, Toshiaki Minami \\ Department of Anesthesiology, Osaka Medical College, Takatsuki, Osaka, Japan \\ ane052@osaka-med.ac.jp,ane060@osaka-med.ac.jp,ane022@osaka-med.ac.jp
}

Keywords: Baroreflex sensitivity, Cardiac-vagal nerve reflex, Head-down tilt

\section{TO THE EDITOR}

Autonomic nervous system activity can be monitored by analyzing the relationship between spontaneous fluctuations of blood pressure and heart rate, known as the baroreflex. The clinical significance of baroreflex sensitivity (BRS) has been reported [1]. However, physiological alterations in BRS under general anesthesia have not been evaluated. We report the effect of steep head-down tilt with pneumoperitoneum on BRS during robot-assisted laparoscopic prostatectomy (RALP). We studied six RALP patients (ASA 1-2, age $68 \pm 5$ years). We calculated BRS using MemCalc/Tonam2C ${ }^{\text {TM }}$ (GMS, Tokyo, Japan); the correlation coefficient for systolic blood pressure and R-R interval was $\geq 0.80$ (Fig. 1). BRS in the horizontal position and in the steep head-down tilt position with pneumoperitoneum during RALP was compared in each patient (Fig. 2). Four out of six patients had a significantly lower BRS in the tilted position than in the horizontal position (Mann-Whitney U-test, $\mathrm{P}<0.05$ ). BRS was 6.0 $\pm 3.3 \mathrm{msec} / \mathrm{mmHg}$ when horizontal, and $3.7 \pm 2.6 \mathrm{msec} / \mathrm{mmHg}$ when tilted. Steep head-down tilt with pneumoperitoneum might therefore influence the autonomic nervous system.

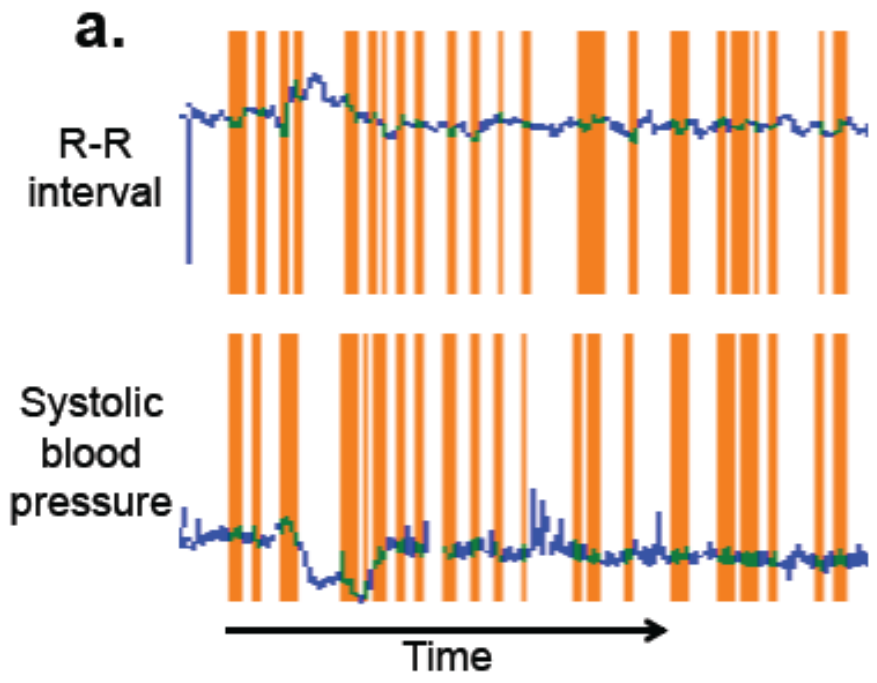

A. Presence of the baroreflex sequence 
Initial studies reported that volatile agents and propofol depressed reflex responses [2]. As anesthetic agents have improved, studies using recent agents are needed. BRS could potentially be used to evaluate the cardiac autonomic nervous activity. This could aid in management during anesthesia, and also in developing strategies for improving long-term outcomes.

The blue lines show the recorded data of the R-R interval and systolic blood pressure. The orange bands show the presence of baroreflex sequences. Baroreflex sequences were defined by at least four consecutive beats in which the systolic blood pressure and R-R interval of the following beat fluctuated by at least $1 \mathrm{mmHg}$ and $1 \mathrm{msec}$, respectively.

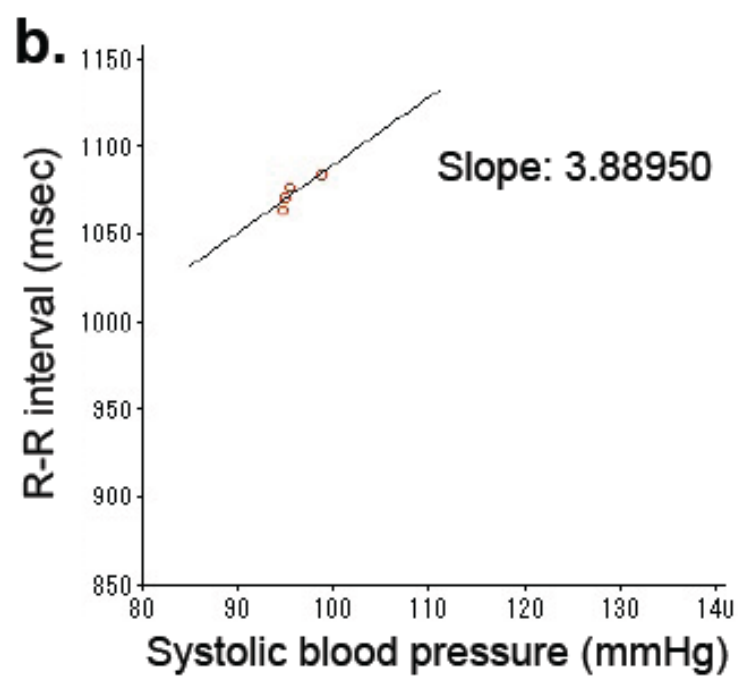

B. A representative of the increasing baroreflex sequence and its sensitivity.

Linear regression was applied to each selected sequence. The slope shown is one of the up-sequences. The mean slope was determined as the average of all slopes, and is considered to be an index of baroreflex sensitivity.

Figure1. Baroreflex sensitivity measurement

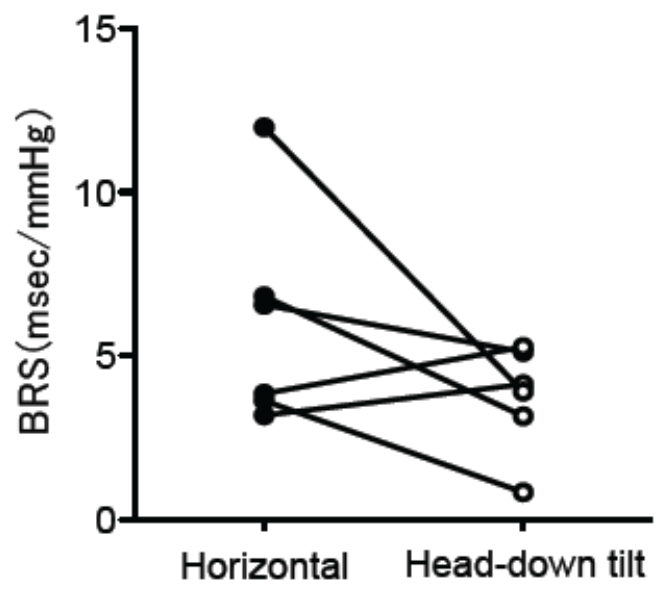

Figure2. Baroreflex sensitivity in the horizontal position and in the steep head-down tilt position during robot-assisted laparoscopic prostatectomy. 
Four of six patients had significantly reduced baroreflex sensitivity in the steep head-down tilt position (white circles) compared with that in the horizontal position (solid black circles).

\section{REFERENCES}

[1] La Rovere MT, Bigger JT Jr, Marcus FI, Mortara A, Schwartz PJ.: Baroreflex sensitivity and heart-rate variability in prediction of total cardiac mortality after myocardial infarction. ATRAMI (Autonomic Tone and Reflexes After Myocardial Infarction) Investigators; Lancet. 1998 Vol.351, No.9101; PP 478-484

[2] Ebert TJ, Harkin CP, Muzi M.: Cardiovascular responses to sevoflurane: a review; Anesth Analg. 1995 Vol.81. No.6; PP S11-22 